\title{
Geometrical Effect in 2D Nanopores
}

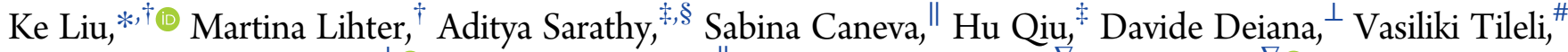 \\ Duncan T. L. Alexander, ${ }^{\perp}{ }^{\perp}$ Stephan Hofmann,, Dumitru Dumcenco, ${ }^{\nabla}$ Andras Kis, ${ }^{\nabla}$ \\ Jean-Pierre Leburton, ${ }^{\ddagger, \S, \odot}$ and Aleksandra Radenovic ${ }^{*}, \dagger$ (i)
}

\begin{abstract}
${ }^{\dagger}$ Laboratory of Nanoscale Biology, Institute of Bioengineering, School of Engineering, ${ }^{\perp}$ Centre Interdisciplinaire de Microscopie Électronique (CIME), " Institute of Materials, ${ }^{\nabla}$ Laboratory of Nanoscale Electronics and Structures, Institute of Electrical Engineering and Institute of Materials Science and Engineering, School of Engineering, EPFL, 1015 Lausanne, Switzerland

${ }^{\ddagger}$ Beckman Institute for Advanced Science and Technology, ${ }^{\S}$ Department of Electrical and Computer Engineering, ${ }^{\bullet}$ Department of Physics, University of Illinois, Urbana, Illinois 61801, United States

"Department of Engineering, University of Cambridge, JJ Thomson Avenue, CB3 0FA Cambridge, United Kingdom
\end{abstract}

\section{Supporting Information}

ABSTRACT: A long-standing problem in the application of solidstate nanopores is the lack of the precise control over the geometry of artificially formed pores compared to the well-defined geometry in their biological counterpart, that is, protein nanopores. To date, experimentally investigated solid-state nanopores have been shown to adopt an approximately circular shape. In this Letter, we

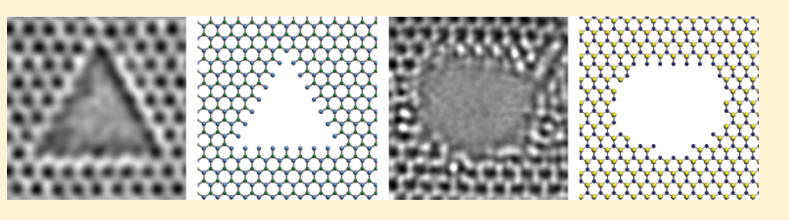
investigate the geometrical effect of the nanopore shape on ionic blockage induced by DNA translocation using triangular h-BN nanopores and approximately circular molybdenum disulfide $\left(\mathrm{MoS}_{2}\right)$ nanopores. We observe a striking geometrydependent ion scattering effect, which is further corroborated by a modified ionic blockage model. The well-acknowledged ionic blockage model is derived from uniform ion permeability through the $2 \mathrm{D}$ nanopore plane and hemisphere like access region in the nanopore vicinity. On the basis of our experimental results, we propose a modified ionic blockage model, which is highly related to the ionic profile caused by geometrical variations. Our findings shed light on the rational design of $2 \mathrm{D}$ nanopores and should be applicable to arbitrary nanopore shapes.

KEYWORDS: Solid-state nanopores, 2D materials, molybdenum disulfide $\left(\mathrm{MoS}_{2}\right)$, hexagonal boron nitride ( $h$-BN), high-resolution transmission electron microscopy (HRTEM), ion transport

$\mathrm{N}$ anopores formed in 2D materials can be considered as apertures that shape ion fluxes through their small volumes. $^{1-5}$ Typical nanopores, reported so far, exhibit geometrical shapes that can be approximated to that of a circle having perfect symmetry. ${ }^{6}$ Such nanopores have been made in a top-down manner, e.g., electron beam sputtering, ${ }^{7-9}$ dielectric breakdown, ${ }^{10,11}$ or electrochemical etching. ${ }^{12}$ So far, there has been no report about the precise control over the geometry of solid-state nanopores. Interestingly, a unique property of hexagonal boron nitride (h-BN) few layers has been revealed by previous transmission electron microscopy (TEM) studies, where triangular defects or nanopores can be created or enlarged while maintaining their intrinsic triangular shape. ${ }^{13-15}$ However, there is no clear understanding about the intrinsic defects in pristine single-layer h-BN, which has a significant influence on ion transport.

Ion transport through atomically pierced $2 \mathrm{D}$ membranes has drawn much attention. ${ }^{16}$ Understanding ion scattering inside nanopores is of vital importance to single molecule resistive pulse sensing; ${ }^{17}$ however, it has been overlooked in the past. It is not clear if continuum theories describing the ion flux in bulk can be directly applied to the systems whose dimensions are comparable to the dimensions of ions. ${ }^{5,18}$ Protein ion channels have well-defined 3D geometries, which facilitate their biological functions. ${ }^{19}$ More recently, single molecule DNA sequencing has been achieved using mutated protein nanopores together with polymerase motor. ${ }^{20}$ Later on, we demonstrated the first example of identifying single-nucleotides in single-layer $\mathrm{MoS}_{2}$ nanopores. ${ }^{21}$ However, due to the lack of the control over the geometry of the nanopores formed in solid-state materials, signal fluctuation stems from such a structural irregularity, which further hinders the high-fidelity DNA sequencing. To design best performing nanopores in the solid-state materials, one needs to gain fundamental understanding about how ions transport through the channels having different geometries. Here, we report the first experimental and theoretical results on such a crucial, yet overlooked phenomenon. We observe a striking geometry-dependent ion scattering effect, which is further corroborated by a modified ionic blockage model. This model complements the wellacknowledged conductance models ${ }^{22,23}$ describing the ions flux inside the nanopore confinement by introducing a factor for the relative ion resistivity inside the pore. Our findings shed light

Received: March 14, 2017

Revised: June 7, 2017

Published: June 8, 2017 

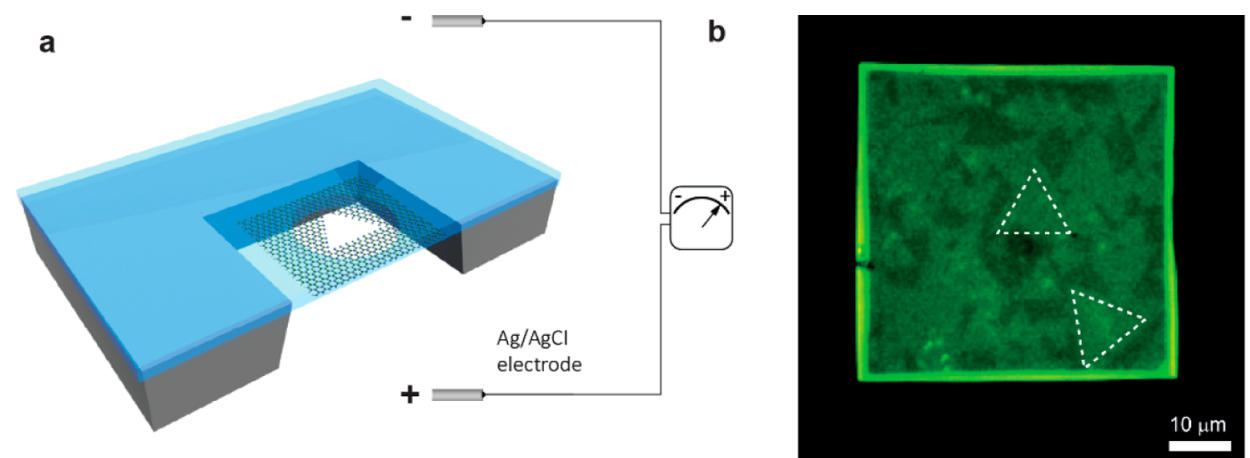

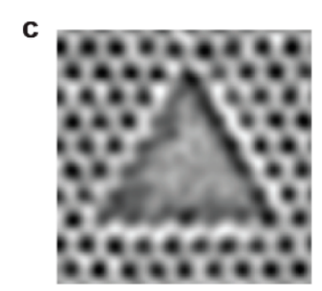

g

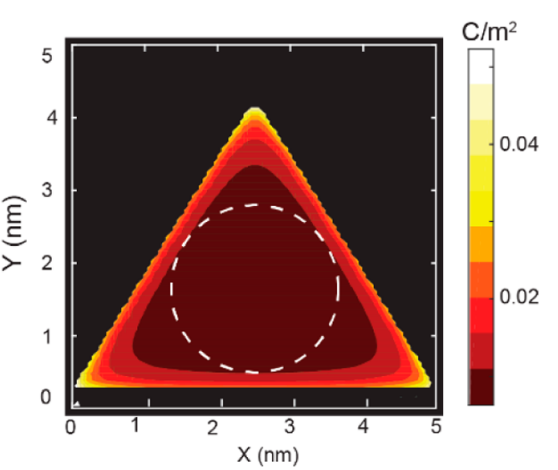

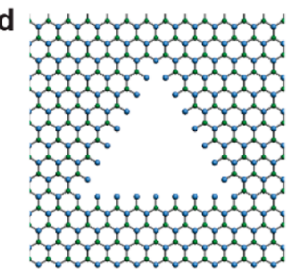

h

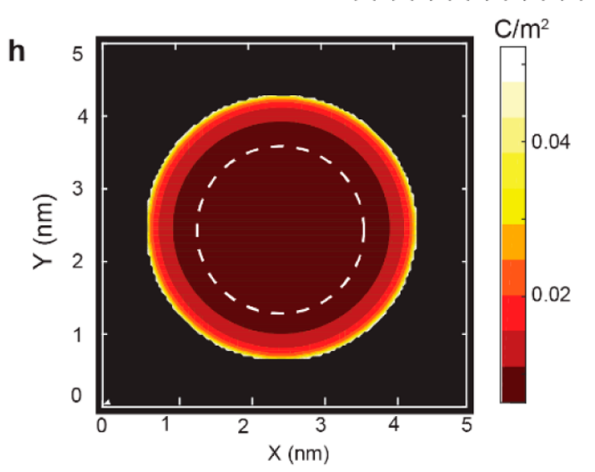

Figure 1. (a) A schematic illustration of a triangular h-BN nanopore membrane, where single-layer h-BN is suspended over an aperture in the SiN ${ }_{x}$ membrane. The device is mounted between two reservoirs filled with saline solution connected to a current amplifier to apply transmembrane bias across the membrane. (b) A bright-field optical image of as-transferred single-layer h-BN over the supporting SiN membrane. Although the observed optical contrast is weak, one can still identify the presence of $\mu \mathrm{m}$-sized triangular h-BN single layer crystals. (c) HRTEM image (taken at $80 \mathrm{kV}$ and with the sample at $400{ }^{\circ} \mathrm{C}$ ) of a triangular h-BN nanopore with white-atom contrast, where atoms are represented by bright spots in a honeycomblike arrangement. (d) 2D lattice representation of the nanopore in single-layer h-BN, where $\mathrm{B}$ atoms are green and $\mathrm{N}$ atoms are gray. The nanopore is terminated by $\mathrm{N}$ atoms. (e) HRTEM image (taken at $80 \mathrm{kV}$ and with the sample at $400{ }^{\circ} \mathrm{C}$ ) of an approximately circular $\mathrm{MoS}_{2}$ nanopore with white-atom contrast, where atoms are represented by bright spots in a honeycomb like arrangement. (f) Corresponding lattice representation of single-layer $\mathrm{MoS}_{2}$, where Mo atoms are purple and S atoms are yellow. The nanopore is terminated by Mo-atoms. (g and h) Contour plots of the charge density distribution along the triangular plate and the circular plate, respectively. Both plates have the same area. The area occupied by dsDNA with a diameter of $2.4 \mathrm{~nm}$ is shown by the dashed circle in $\mathrm{g}$ and $\mathrm{h}$.

on the rational design of $2 \mathrm{D}$ nanopores and should be applicable to arbitrary nanopore shapes. With this work, we provide a possible roadmap, first computationally to build optimal nanopore geometry, and later experimentally explore the possibilities of building such a nanopore.

Figure 1a shows a schematic illustration of our nanopore device. Similarly to other nanopores made of $2 \mathrm{D}$ material, ${ }^{1,4}$ a single-layer of h-BN, grown by CVD (chemical vapor deposition), is suspended over a small opening in a $\mathrm{SiN}_{x}$ membrane. The transferred h-BN domains can be easily identified by optical microscopy (Figure 1b) due to their characteristic triangular shape. ${ }^{24,25}$ Further details on the growth, transfer, and fabrication process can be found in SI and Figure S1. Interestingly, not only do the h-BN domains $(\sim 10 \mu \mathrm{m}$ side length) grow as triangular islands, but the atomic-scale defects within the lattice also adopt a characteristic triangular shape. Such defects in single-layer h-BN can be activated either by heating or by electron beam irradiation. ${ }^{26}$ Figure 1c shows a high-resolution TEM (HRTEM) image of a typical triangular nanopore in single-layer h-BN, with nitrogen (N)-terminated edges, ${ }^{13-15}$ while Figure $1 \mathrm{~d}$ illustrates the atomic arrangement of its defective h-BN lattice. The topological rigidity of such defects is related to the selective removal of the atoms next to a vacancy or at the edge of a large hole compared to the atoms in the pristine lattice. This phenomenon has already been observed primarily by TEM under electron beam irradiation. According to the previous TEM studies, two-coordinated $\mathrm{N}$ atoms have a much lower ejection probability than two-coordinated boron (B) atoms, which results in a zigzag removal of atoms along the defect edge. ${ }^{15}$ Remarkably, during the entire irradiation process, the pore shows a propensity to adapt a triangular shape, which we also observe and discuss later in more detail. In contrast, the $\mathrm{MoS}_{2}$ nanopore adopts an approximately circular geometry (Figure 1e), as schematically illustrated in Figure 1f, since, in the $\mathrm{MoS}_{2}$ lattice, there is a tendency toward random etching or knock-on displacement of atoms under electron beam irradiation. From the energetic perspective, the final geometry 

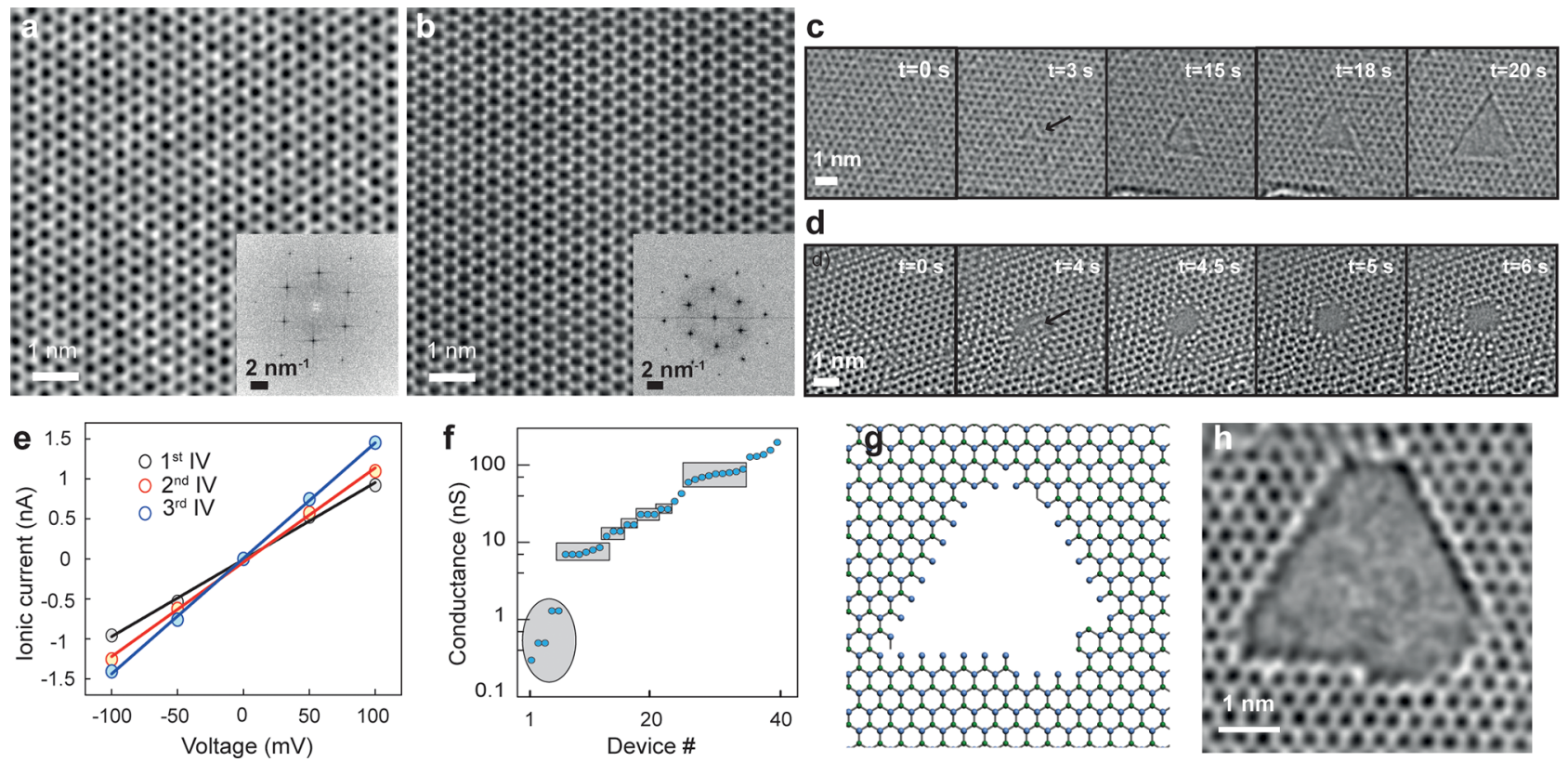

Figure 2. (a) HRTEM image of pristine h-BN lattice. Inset: diffractogram corresponding to the area in a), with a secondary order reflections visible. (b) HRTEM image of pristine $\mathrm{MoS}_{2}$ lattice of the same size as in panel a, $8 \mathrm{~nm} \times 8 \mathrm{~nm}$. Inset: corresponding diffractogram with second and third order reflections clearly visible. (c) Time-lapse HRTEM images of a triangular h-BN nanopore evolving in size while still maintaining its triangular shape. The elapsed time is marked on each image. (d) Time-lapse HRTEM images of an approximately circular $\mathrm{MoS}_{2}$ nanopore evolving in size. The elapsed time is marked on each image. (e) Consecutive $I V s$ in a small h-BN nanopore showing gradual growth. (f) Initial conductances measured in $1 \mathrm{M} \mathrm{KCl}$ of all working devices in the study. (g) Illustration of partial removal of atoms along the N-terminated edge of an h-BN nanopore. (h) HRTEM image corresponding to the sketch in $\mathrm{g}$. All of the TEM images were taken at $80 \mathrm{kV}$ and with the sample at $400{ }^{\circ} \mathrm{C}$ except in panels $\mathrm{c}$ and $\mathrm{d}$ which were taken at $80 \mathrm{kV}$ and with the sample at room temperature.

of such a formed $\mathrm{MoS}_{2}$ nanopore will have a circular shape. Interestingly, orientation preference of defects has not yet been observed for any $2 \mathrm{D}$ materials other than $\mathrm{h}-\mathrm{BN}$, which makes this material a good candidate for nanopore sensing. To understand the ionic flow profiles inside these two types of nanopores with different symmetries, we use the method of moments $(\mathrm{MoM})^{27,28}$ to calculate the total capacitance $(C)$ of a triangular and circular conducting plate (the details can be found in the SI). These calculations allow us to obtain the charge distribution along the plate, where we observe that in the case of triangular nanopores in $\mathrm{h}-\mathrm{BN}$, the edges, and especially the corners, display large variations in charge density distribution (Figure 1g).

We utilized TEM to inspect further the nanoscopic features of single-layer h-BN and $\mathrm{MoS}_{2}$. To minimize the beam induced knock-on damage of the monolayer, we exploited an aberrationcorrected (with double Cs corrector) FEI Titan Themis 60-300 TEM instrument operated at either $80 \mathrm{kV}$ or $60 \mathrm{kV}$ acceleration voltage. For HRTEM imaging the incident electron beam was monochromated to reduce the effects of chromatic aberration, thereby improving contrast from light $\mathrm{B}$ and $\mathrm{N}$ atoms for adequate image interpretation. The low-magnification image in Figure S2a shows a suspended area of the h-BN covering a $\mathrm{SiN}_{x}$ aperture, where only a little trace of PMMA residue can be seen. Special care should be taken when cleaning the PMMA to obtain atomically resolved HRTEM imaging as well for high sensitivity nanopore sensing. An HRTEM image of the h-BN lattice can be seen in Figure 2a, where the lattice is represented by a white-atom contrast ${ }^{29,30}$ that is directly related to actual atomic positions. While the contrasts of $\mathrm{B}$ and $\mathrm{N}$ atoms are similar, they are distinguishable under suitable imaging conditions. The inset shows the fast Fourier transform (FFT) of this perfect lattice, confirming its crystalline structure with hexagonal symmetry. Besides the first set of reflections, from which we can derive the h-BN lattice constant of $2.5 \AA$, the second set of reflections corresponds to the $1.4 \AA$ separation of $\mathrm{B}-\mathrm{N}$ atoms. Figure S3 shows examples of HRTEM imaging of h-BN represented in black-atom contrast, where instead the edge atom arrangement and point defects cannot be readily interpreted. For comparison, an HRTEM image of the $\mathrm{MoS}_{2}$ lattice is shown in Figure $2 \mathrm{~b}$ with its corresponding FFT (inset), revealing the characteristics of the lattice in reciprocal space.

As mentioned above, a unique feature of $\mathrm{h}-\mathrm{BN}$ compared to other 2D materials (graphene, $\mathrm{MoS}_{2}$, etc.) is that its defects can maintain triangular shapes. Figure $2 c$ shows time-lapse frames (frames acquired with an exposure time of $0.1 \mathrm{~s}$ ) depicting a triangular h-BN nanopore evolving in size under electron beam irradiation (Movie S1). This is supported by the TEM image in Figure S2b, where, after the chains of $\mathrm{B}$ and $\mathrm{N}$ atoms are subsequently removed along the edges in a zigzag manner, the triangular defect enlarges to a size greater than $10 \mathrm{~nm}$ in side length. Conversely, $\mathrm{MoS}_{2}$ adopts a less regular, approximately circular shape during electron beam irradiation, as shown in the time-lapse frames (/0.1 s) in Figure 2d. During the prolonged irradiation, we observe another interesting behavior of $h-B N$, where adjacent small triangular nanopores can merge to form a larger triangular nanopore, as displayed in Figure S2c and Figure S2d. This is due to the high mobility of the B and N atoms under electron beam irradiation which favors their rearrangement into more stable configurations. However, due to the fast dynamics of defect growth under electron beam exposure, it is extremely challenging to visualize the pristine h$\mathrm{BN}$ defects (i.e., defects present from the growth stage/ 
transfer). We therefore exploited specimen heating capability in TEM in order to preserve the pristine nanopore geometry during imaging. ${ }^{31}$ As shown in Movie S2, at a high specimen temperature and during a longer observation time ( $2 \mathrm{~s})$, the triangular defects do not show significant growth. In this way, we were able to verify that the defects in as-transferred singlelayer h-BN also adopt a triangular shape. Moreover, we were able to measure the density of pristine h-BN defects by using a MAADF (medium-angle annular dark-field)-scanning (S)TEM imaging condition (i.e., a low acceleration voltage of $60 \mathrm{kV}$ and a low probe current of $30 \mathrm{pA}$ ) combined with specimen heating, albeit with a trade-off of reduced atomic contrast in the h-BN lattice compared to the HRTEM imaging. As shown in Figure S4, we found a low defect density $\left(0.001 \mathrm{~nm}^{-2}\right)$, which we believe comes inherently from the growth or the transfer steps.

After TEM characterization, we focus on the use of h-BN as 2D membrane for nanopore sensing. Current-voltage (IV) characteristics give direct feedback of the pore formation and the related pore size. It is worth noting that a small $\mathrm{hBN}$ nanopore is quite stable during experiments which can be seen in the example of sequential $I V$ measurements given in Figure $2 \mathrm{e}$, resulting in $0.2 \mathrm{~nm}$ enlarging of the pore size during $2 \mathrm{~h}$. Although these devices were not exposed to electron beam, we found that only $20 \%$ of successfully transferred samples had the conductance $<1 \mathrm{nS}$ which we consider as an intact h-BN membrane. We assume that the remaining $80 \%$ of the devices already had a certain level of defects. From a large statistical data set, we found a discrete trend in the conductance spanning over a large range as shown in Figure $2 \mathrm{f}$. Here, we only used the first set of $I V$ to calculate the initial open pore conductance. We can tentatively attribute this discontinuity to certain energetically favorable sizes, which we do not observe in other $2 \mathrm{D}$ nanopores. The major plateaus in the conductance distribution corresponds to triangular defects in the h-BN lattice with different lengths. As shown in Figure 2g, we assume that the triangular defect is $\mathrm{N}$-terminated and possesses several unstable dangling $\mathrm{N}$ atoms along the triangle edge marked by the dashed circles, giving rise to minor modulations in the conductance. The partial removal along the $\mathrm{N}$-terminated edges can be seen in the Figure $2 \mathrm{~h}$.

In the case of a defect-free h-BN membrane, an electrochemical reaction (ECR) method ${ }^{12}$ (previously used for $\mathrm{MoS}_{2}$ nanopore formation) is used to create a nanopore in h-BN by applying trans-membrane voltage higher than the local threshold potential to remove $\mathrm{B}$ and $\mathrm{N}$ atoms. Figure S5a shows a representative ECR current trace to demonstrate the pore formation process, where a nanopore is formed at $3 \mathrm{~V}$. The pore growth manifests itself in a continuous increase in the ionic current. Due to the relatively high ECR threshold voltage of $3 \mathrm{~V}$, it is challenging to observe single atom nanopore opening as recently reported for $\mathrm{MoS}_{2} .{ }^{12}$ The threshold bias observed here in the liquid/solid junction is in good agreement with a previous STM study. ${ }^{32}$ Figure S5b shows the $I V$ characteristics of this device before and after the ECR process with a clear transition from an insulating, pore-free h-BN membrane to a porous $\mathrm{h}-\mathrm{BN}$ membrane containing a single nanopore. In this work we focus on the effects that a welldefined nanopore geometry has on the conductance and biomolecular sensing; therefore, we do not use any ECRformed nanopores as we cannot directly assess the resulting pore geometry.
To test the sensing capability of a triangular h-BN nanopore, we used a device already having a $48 \mathrm{nS}$ open pore conductance in $1 \mathrm{M} \mathrm{KCl}$. We injected $1 \mathrm{k} \mathrm{bp} \mathrm{dsDNA}$ into the cis-side of the flowcell and applied positive potential on the trans-side to drag the negatively charged DNA passing through the pore. As expected, transient blockages are observed in short experiment time (Figure 3a). The power spectral density (PSD) in Figure

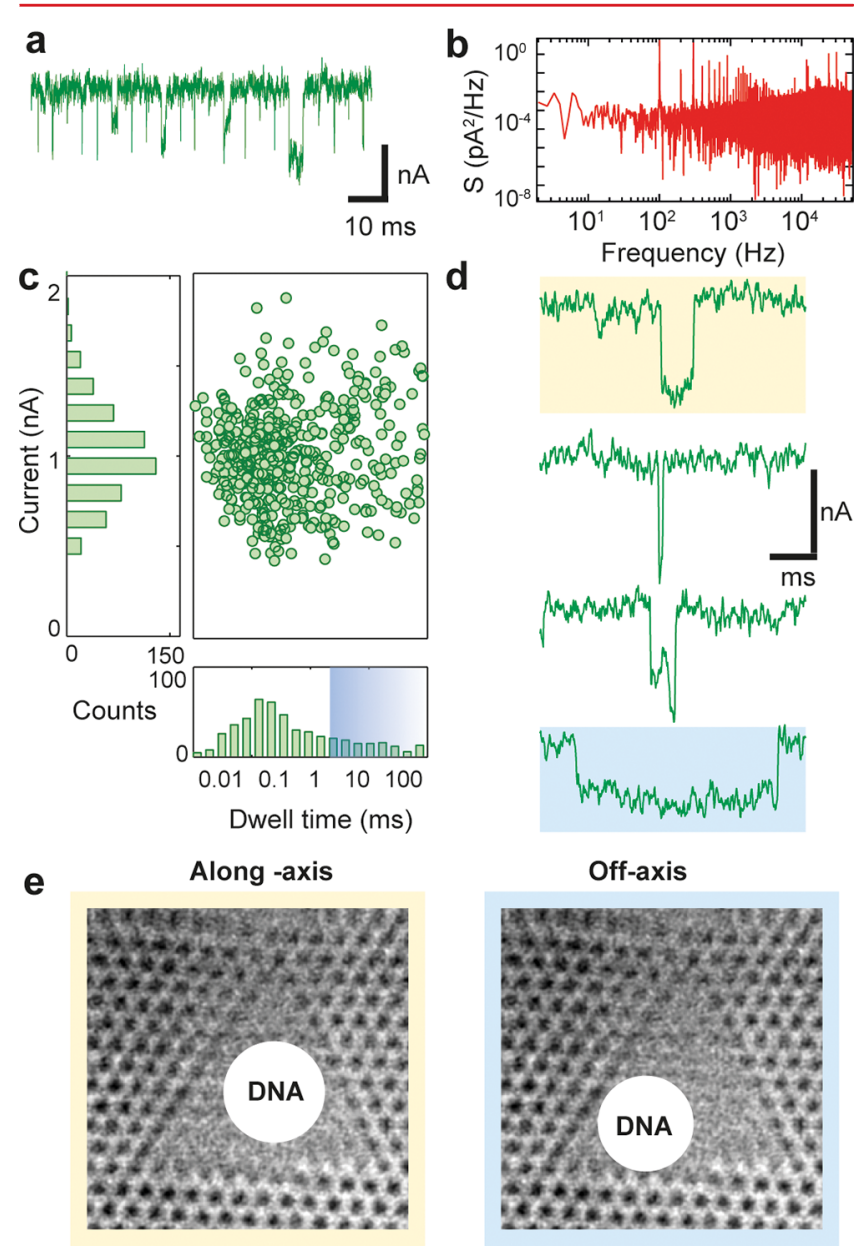

Figure 3. (a) A current trace of concatenated events in an h-BN nanopore with a $48 \mathrm{nS}$ open pore conductance in $1 \mathrm{M} \mathrm{KCl}$. The event detection is performed using an open source OpenNanopore software. (b) Power spectral density (PSD) of a short fragment of open pore current trace that depicts the frequency dependent noise performance of the device. The trace is recorded at $0 \mathrm{mV}$ with ionic strength of $1 \mathrm{M}$ $\mathrm{KCl}$. RMS noise is $\sim 35 \mathrm{pA}$ at 10k lowpass filtering. (c) Scatter plot of current drop versus dwell time of detected events at $400 \mathrm{mV}$. (d) Representative events showing linearized smooth translocation, folded fast translocation, partially folded translocation, and retarded translocation, respectively. (e) Plausible explanations for retarded translocation due to sterically hindered DNA motion by the pore geometry.

3b exhibits a similar noise performance compared to $\mathrm{MoS}_{2}$ nanopores. RMS noise is $\sim 35 \mathrm{pA}$ at $10 \mathrm{k}$ lowpass filtering, which is comparable to $\mathrm{MoS}_{2}$ nanopores. ${ }^{12,33}$ The major contribution is low frequency $1 / f$ noise, ${ }^{34}$ which can be reduced significantly by shrinking the region of suspended h-BN to a circular aperture with a diameter of $50 \mathrm{~nm}$. In this way, we obtain a very good signal-to-noise ratio (SNR) which is up to 20. Figure $3 c$ shows a scatter plot of translocation events recorded at $400 \mathrm{mV}$ with some representative events selected displayed in Figure 3d. As can be observed in Figure S6, the 
amplitudes are proportional to applied bias, indicating a voltage-driven process. Quantitatively, $0.47 \pm 0.18 \mathrm{nA}, 0.67 \pm$ $0.21 \mathrm{nA}$, and $1.03 \pm 0.34 \mathrm{nA}$ are found for $200 \mathrm{mV}, 300 \mathrm{mV}$, and $400 \mathrm{mV}$, respectively. Moreover, while the amplitude at each voltage displays unimodal distribution, the dwell time shows bimodal distribution. The first distribution which is in the fast translocation regime is consistent with a free translocation time for $1 \mathrm{k} \mathrm{bp}$ length DNA, which is in the range of $\sim 100 \mu \mathrm{s}$. Interestingly, the second distribution in the slow translocation regime is in the range of 5-100 ms, indicating that a retardation mechanism governs the translocation dynamics. In the previous reports, slow translocations are in the long tail of the dwell time distribution. ${ }^{35-37}$ Here, we suggest a geometrical retardation mechanism stemming from the unique triangular shape of h-BN nanopores. As sketched in Figure $3 \mathrm{e}$, we suggest that the movement along the vertical axis and off-axis movement are responsible for fast translocation and slow translocation, respectively. It is easy to understand that a translocated DNA molecule experiences high steric hindrance when it moves off-centered which could be the major reason for the observed large population of slow translocation events. In larger h-BN nanopores, we observe significantly fewer slow translocations.

To further explore the origin of the fast and slow translocation, we performed molecular dynamics simulations of a dsDNA molecule translocating ${ }^{38,39}$ through a BN nanopore along the on-axis (left) and off-axis (right) paths, respectively. For this purpose, a mathematical boundary perpendicular to the $\mathrm{BN}$ sheet was applied to constrain the lateral movement of the DNA around the BN sheet plane (Figure 4a). It was found that

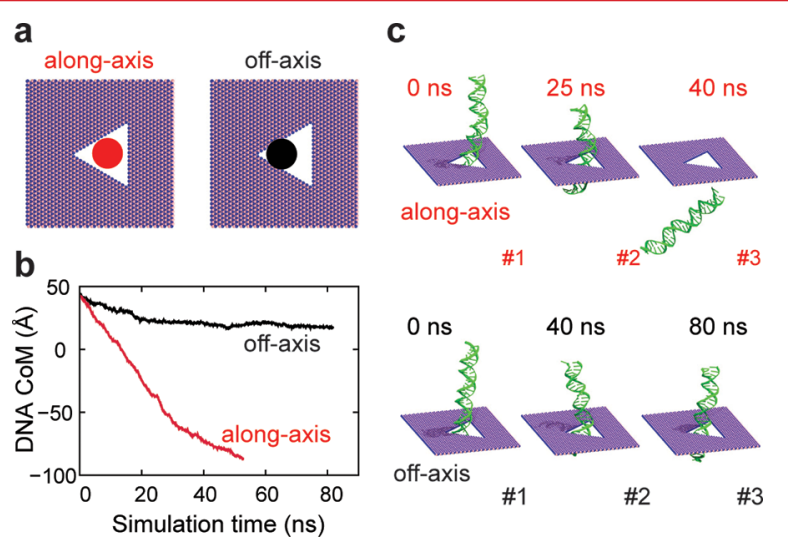

Figure 4. Molecular dynamics simulations of a dsDNA molecule translocating through an h-BN nanopore. (a) Two mathematical boundaries perpendicular to the $\mathrm{h}-\mathrm{BN}$ sheet were applied in our MD simulations to ensure the DNA movement through the pore along the along-axis (left) and off-axis (right) paths, respectively. (b) Change of the center of the mass (CoM) of the DNA molecule with respect to simulation time. (c) Snapshots taken from MD simulations of the along-axis (top) and off-axis (bottom) DNA translocation through the h-BN nanopore.

the DNA permeates through the pore along the vertical axis much faster than off-axis, in good agreement with our above speculation (Figure 4b). Further analysis suggests that the slow off-axis translocation is the consequence of the strong interaction between the $\mathrm{h}-\mathrm{BN}$ pore edge and the dsDNA (Figure 4c). The nature of these interactions between the DNA and triangular h-BN nanopore is due to van-der Waals and electrostatic forces, which are taken into account in the force field parameters of the MD simulations. This is further outlined in section 6 of the SI.

Another striking finding is that the observed current blockages in h-BN are consistently lower than the values in $\mathrm{MoS}_{2}$ nanopores with a comparable open pore conductance. To obtain more detailed information about the impact of nanopore size and geometry on the conductance, we decided to use dsDNA as a molecular ruler. For each experimental DNA translocation data set, we plotted the $\Delta G_{\text {relative }}$ vs $G_{\text {open }}$ where $\Delta G_{\text {relative }}$ is a relative conductance drop given by $\left(G_{\text {open }}-\right.$ $\left.G_{\text {blocked }}\right) / G_{\text {open }}$ while $G_{\text {open }}$ and $G_{\text {blocked }}$ are the conductances for an open pore and pore blocked by DNA, respectively. Because of notable spreading of translocation data, we defined different event clusters by using a Gaussian Mixture Distribution fitting where each cluster is represented with a single center point and a standard deviation in $x$ and $y$. More information can be found in the SI and in Figure S8. The resulting data was then used to develop a conductance model which provides more insights into the ionic and DNA transport through triangular nanopores.

The well-acknowledged conductance model ${ }^{22,23}$ that describes the passage of ions through the nanopore includes two terms, which are the channel resistance (the nanopore itself) and the access resistance ${ }^{40}$ (the vicinity of the nanopore). Surfaces charges of h-BN and $\mathrm{MoS}_{2}$ are similar and play a significant role at low ionic strength $(10 \mathrm{mM}) .{ }^{41}$ In this work we focus on the geometrical effect, therefore, we tried to minimize the surface charge influence by using a solution of high ionic strength $(1 \mathrm{M})$. Equation 1 gives the analytic expression of this model, where $\sigma, L$, and $d$ are the ionic conductivity of the solution $(10.5 \mathrm{~S} / \mathrm{m}$ for $1 \mathrm{M} \mathrm{KCl}$ at 23 $\left.{ }^{\circ} \mathrm{C}\right){ }^{23}$ the effective membrane thickness (1.6 and $1.4 \mathrm{~nm}$ for $\mathrm{MoS}_{2}$ and h-BN, respectively, accounting for the hydrodynamic effect), ${ }^{4,42}$ and the equivalent nanopore diameter for a perfect circle, respectively.

$$
G=\sigma_{\text {bulk }}\left[\frac{4 L}{\pi d^{2}}+\frac{1}{d}\right]^{-1}
$$

This model assumes that the ion mobility inside the nanopore is identical to the bulk mobility without restraint. For typical circular-shaped nanopores, the channel effect and the access effect are equally dominant due to the perfect symmetry of the circular shape. However, a triangular h-BN nanopore has 3-fold symmetry, giving rise to a nonuniformity in the ion distribution inside and in the vicinity of the nanopore. This distribution of the ion flux within the pore for different pore shapes, studied using MD simulations, is outlined in section 6 of the SI. It is worth noting that few reports have discussed geometrical effects due to the difficulty in the fabrication methods. ${ }^{43,44}$ The modifications of the conductance model are mainly based on the $z$-axis variation perpendicular to the nanopore plane. In our case, two important factors should be reconsidered due to geometry or symmetry breaking, which are the calculation of the capacitance determined by the spatial charge density distribution and the relative ion conductivity inside the nanopore. Both factors are highly dependent on the geometry. Here, we can make two mutually exclusive assumptions, one where the intrinsic pore resistance is dominant and the other where, conversely, the access resistance dominates. Both models have only a single unknown parameter, while all other parameters can be obtained unambiguously. We first discuss a triangular (equilateral) 
pore, for which the expressions for the pore and access resistances are given by $R_{\text {pore }}=\rho_{\text {pore }}\left(4 L / \sqrt{ } 3 a^{2}\right)$ and $R_{\text {access }}=$ $\rho_{\text {access }}\left(\varepsilon / C_{\Delta}\right)$, respectively, where $L$ is the hydrodynamic thickness of h-BN membrane, $a$ is the edge length of the triangular pore, $\varepsilon$ is the permittivity in free space, and $C_{\Delta}$ is the capacitance of the triangular plate (details of the calculations are available in the SI). $\rho_{\text {pore }}$ and $\rho_{\text {access }}$ are the resistivity of the pore and the access resistivity, respectively, which we assume are different. Indeed, $\rho_{\text {pore }}$ varies from one pore shape to another depending on the scattering of ions by the pore edges and corners. These edge effects contribute to a higher intrinsic resistivity due to the pore shape. Hence, we can relate the intrinsic pore resistivity to the access resistivity through a single factor $\alpha_{\text {pore }}$ which leads us to equation:

$$
G=\sigma_{\text {bulk }}\left[\frac{1}{\alpha_{\text {pore }}} \frac{4 L}{\sqrt{3} a^{2}}+\frac{2 \epsilon}{C_{\Delta}}\right]^{-1}
$$

For the access resistance dominant model, namely the "pseudo circular model", we use a prefactor $k$ to encompass all the information including pore shape, edges, and scattering effects.

$$
G=\sigma_{\text {bulk }}\left[\frac{4 L}{\sqrt{3} a^{2}}+\frac{k}{a}\right]^{-1}
$$

As shown in Figure 5a, the $\alpha$ model produces the best fits (referred to section 8 in the SI) to experimental h-BN data. According to the minimal root-mean-square-error (RMSE) criteria the best fitting is obtained with $\alpha_{\text {pore }}=0.3$, while the $\alpha_{\text {pore }}=0.15$ gives an excellent agreement for the low $\Delta G_{\text {relative }}$ data points except for the highest point at the low $G_{\text {open }}$. The small value of $\alpha_{\text {pore }}$ translates into a high intrinsic resistivity of ions inside the nanopore, which may originate from either low ion concentration, or low ion mobility inside the pore, or both. A more sophisticated approach ${ }^{18}$ is required to address this. The strong discrepancy with the classic model (eq 1) reveals a very interesting nanoscopic shaping of the ion flux inside a triangular nanopore. The pseudo circular $k$ model fits data less well (higher RMSE, referred to section 8 in the SI), while still being able to give a prefactor $k$ of 2.5 , as shown in Figure S9a.

In contrast, the pseudo circular $k$ model fits the $\mathrm{MoS}_{2}$ data slightly better than the $\alpha$ model, shown in Figure S9b. We obtained lower $k$ value (1.1) then in the h-BN case, which shows that the classic model still holds for approximately circular $\mathrm{MoS}_{2}$ nanopores. $\mathrm{MoS}_{2}$ data points look more dispersed comparing to h-BN data set, which could be possibly due to several uncontrollable factors, e.g., deviation from the perfect circular geometry (which would also affect the surface charges in the pore mouth), or locally increased $L$ thickness due to PMMA residues. Therefore, we also show the $\alpha$ model fittings that fit better for certain data points. A small discrepancy is observed if using the modified models, which explains the lack of the knowledge of the overestimated ion conductivity inside the pore. ${ }^{45}$ Compared to the small $\alpha_{\text {pore }}$ value in $\mathrm{h}-\mathrm{BN}$, ions have much higher permeability through $\mathrm{MoS}_{2}$ nanopores, that is clearly visible for the $\mathrm{MoS}_{2}$ points fitted with $\alpha_{\text {pore }}=0.8$ which is close to the bulk value. In other words, the channel resistance plays a major role in sensing molecules in the case of h-BN nanopores; however, it results in a smaller total conductance drop. We also examined the underestimated side length $a$ of the triangular hBN nanopore (Figure $5 \mathrm{~b}$ ), which strongly depends on $\alpha_{\text {pore }}$. As we already mentioned, the cleanness of the $2 \mathrm{D}$ material is of high
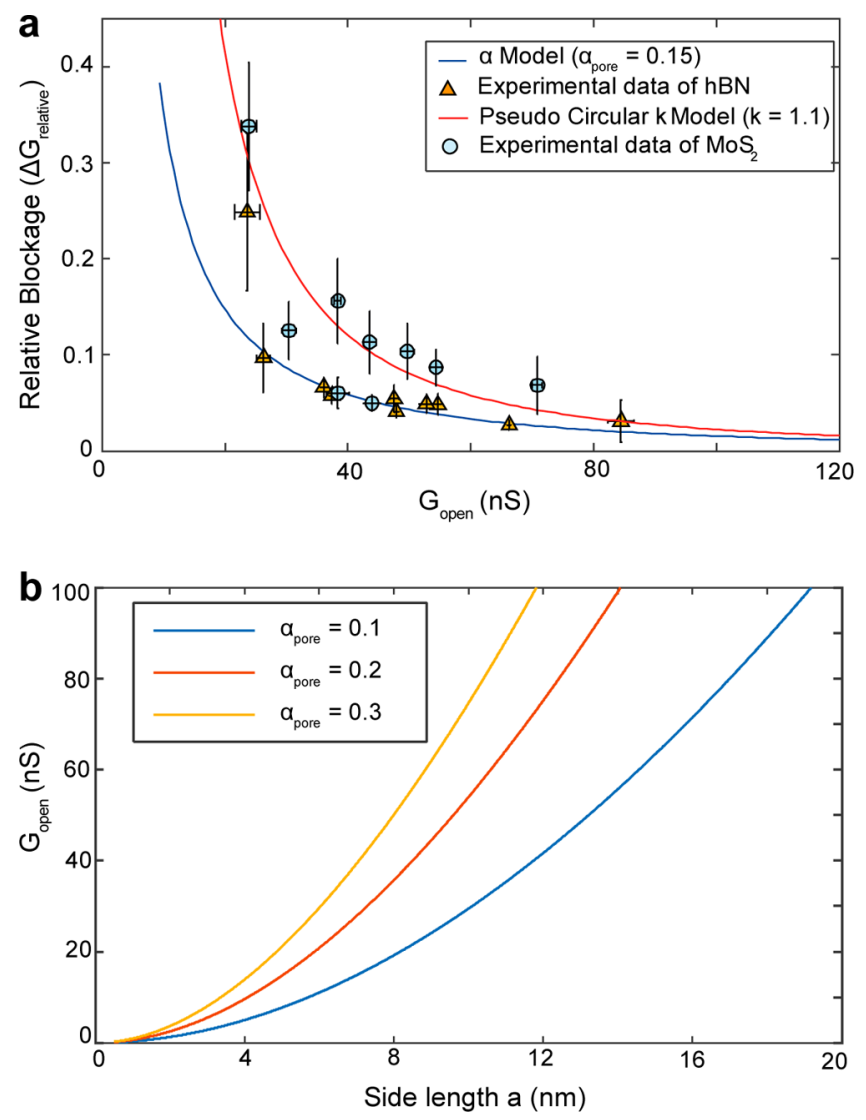

Figure 5. (a) Comparison of best-fitting theoretical models with experimental data. h-BN experimental data are best fit to $\alpha$ model, where $\alpha_{\text {pore }}$ is the proportional factor of ion conductivity inside the pore. $\mathrm{MoS}_{2}$ experimental data are best fit to pseudo circular $k$ model, where $k$ is the corrected factor for the access resistance. More details are in the SI section 8 . All translocation data were taken using dsDNA ( $1 \mathrm{k} \mathrm{bp}, 5 \mathrm{k} b p$, and $48 \mathrm{k} \mathrm{bp}$ ). (b) Dependence of calculated side length $a$ of the triangular hBN nanopore on $\alpha_{\text {pore }}$.

importance since the polymer residues can locally affect the thickness and morphology of the pore vicinity and thus influence the conductance and DNA-pore interaction. We tried to avoid this issue by rigorous cleaning procedure of $h-B N$ and $\mathrm{MoS}_{2}$ after the transfer; however, to have clean polymerfree membranes the best would be to grow 2D material directly over the $\mathrm{SiN}_{x}$ opening, as has been previously shown. ${ }^{33}$

To test if we can exploit the confined geometry of h-BN nanopore to control the entering of molecules into the nanopore, we perform homopolymer translocation through a small $2.5 \mathrm{~nm}$ (side length) h-BN nanopore. To our surprise, we observe sufficient events to make statistics without using a viscous medium. ${ }^{21}$ Figure $6 \mathrm{a}$ shows representative traces of poly $_{30}$ and polyC $\mathrm{C}_{30}$ translocations. Statistically, we are able to differentiate their chemical identities by current drop during translocation, shown in Figure $6 \mathrm{~b}$. Due to the limited bandwidth of the Axopatch current amplifier $(100 \mathrm{k} \mathrm{Hz})$, we speculate that detectable events originate from even more pronounced DNA-pore interaction due to the off-axis movement of short ssDNA molecules through the h-BN nanopore. At the same experimental conditions (pore size and ionic strength), translocations of such short ssDNA cannot be detected in $\mathrm{MoS}_{2}$ nanopores without employing a viscous medium. 


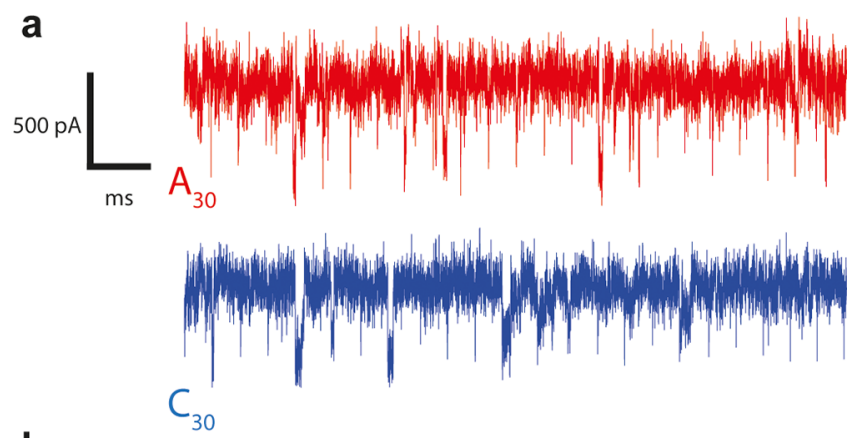

b

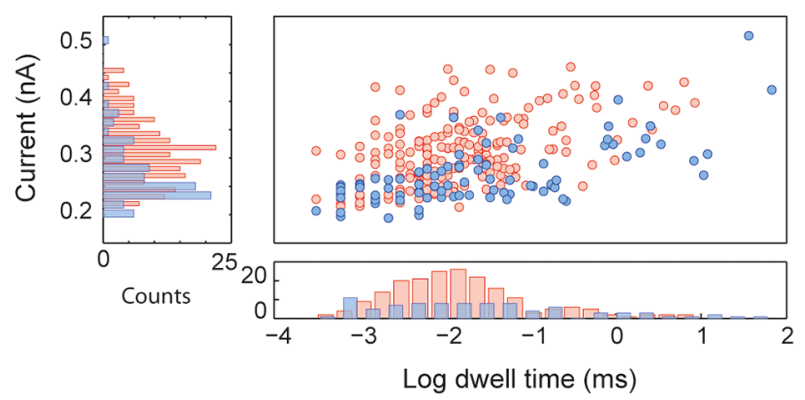

Figure 6. (a) Homopolymer translocation in a small h-BN nanopore. Poly $_{30}$ is shown in red and polyC $\mathrm{C}_{30}$ in blue. (b) Scatter plot of poly $_{30}$ and polyC $\mathrm{C}_{30}$ events.

Top-down engineering of well-defined (in terms of size distributions, defect density, and geometry) defects in 2D materials has a significant role in large scale nanoelectronics and various applications (biosensing, ${ }^{21,46,47}$ desalination, ${ }^{48}$ or osmotic power generation, ${ }^{49}$ etc.). Here we demonstrate the first example, to the best of our knowledge, of geometrydependent ion scattering and therefore single-molecule nanopore biosensing. Owing to the well-defined triangular defects in single-layer h-BN, we tried to provide valuable insights of the ion transport through such geometry experimentally and theoretically. Furthermore, we build a better understanding of the roles of access region and channel region in single-molecule sensing on the basis of a modified conductance model with one fitting parameter $\alpha_{\text {pore }}$, namely, the relative ion resistivity inside the nanopore. This model reveals an anomalous process when the dimensions of the nanopores are reduced to the atomic scale. To design nanopores in $2 \mathrm{D}$ materials in a rational way, one should understand the geometrical effect of the nanopore shape on the ion flux. DNA translocation through $\mathrm{MoS}_{2}$ nanopores exhibits an access resistance dominant process, while DNA translocation through $\mathrm{hBN}$ nanopores exhibits a channel resistance dominant process. DNA induced conductance drop $(\Delta G)$ in $\mathrm{MoS}_{2}$ nanopores is superior to that in h-BN nanopores, due to the high ion flux through $\mathrm{MoS}_{2}$ nanopores. The role of ions in revealing the chemical identities of DNA, i.e., DNA sequencing, ${ }^{17,19}$ is not completely clear. Further study is required to understand the fundamentals of ionic blockage-based DNA nanopore sequencing.

\section{ASSOCIATED CONTENT}

\section{S Supporting Information}

The Supporting Information is available free of charge on the ACS Publications website at DOI: 10.1021/acs.nanolett.7b01091.
Additional information on experimental details (CVD growth, transfer and nanofluidic setup), complementary TEM observations, detailed mathematical calculations, and $\mathrm{MD}$ simulations (PDF)

Formation of a triangular h-BN nanopore under irradiation (AVI)

Stability of a triangular h-BN nanopore under heat (AVI)

\section{AUTHOR INFORMATION}

\section{Corresponding Authors}

*E-mail: ke.liu@epfl.ch. Tel.: +41 21693 1163. Fax: +41 21 6937380.

*E-mail: aleksandra.radenovic@epfl.ch. Tel.: +41 216937371. ORCID $\odot$

Ke Liu: 0000-0002-1976-2297

Duncan T. L. Alexander: 0000-0003-4350-8587

Andras Kis: 0000-0002-3426-7702

Jean-Pierre Leburton: 0000-0002-8183-5581

Aleksandra Radenovic: 0000-0001-8194-2785

\section{Present Address}

H.Q.: State Key Laboratory of Mechanics and Control of Mechanical Structures, Nanjing University of Aeronautics and Astronautics, Nanjing 210016, China.

\section{Author Contributions}

K.L., M. L., and A.R. conceived the idea of comparing geometrical effects in 2D nanopores, designed experiments, proposed modifying the conductance model, and wrote the manuscript. K.L. and M.L. fabricated the nanopore device and performed measurements and data analysis. Q.H. performed molecular dynamics under J.P.L.'s supervision. A.S. and J.P.L. proposed the new model for the size effects and roughness in the nanopore conductance and computed rigorously the nanopore self-capacitance to determine the access resistance. S.C. conceived the idea of using triangular $\mathrm{hBN}$ nanopores, initiated the collaboration, participated at the early stage of the research, and performed CVD h-BN growth under S.H.'s supervision. Du.D. performed CVD $\mathrm{MoS}_{2}$ growth under A.K.'s supervision. Da.D., V.T., and D.T.L.A performed HRTEM imaging. All authors provided constructive comments on the manuscript. K.L. and M.L. contributed equally to this work.

\section{Notes}

The authors declare no competing financial interest.

During the final preparation of the manuscript, we have noticed that a manuscript with similar concept but only TEM results appeared on arxiv online repository (https://arxiv.org/ftp/ arxiv/papers/1702/1702.01220.pdf).

\section{ACKNOWLEDGMENTS}

This work was financially supported by the European Research Council (grant 259398, PorABEL), by a Swiss National Science Foundation (SNSF) Consolidator grant (BIONIC BSCGI0 157802), by SNSF Sinergia grant 147607. We thank the Centre Interdisciplinaire de Microscopie Electronique (CIME) at the École Polytechnique fédérale de Lausanne (EPFL) for access to electron microscopes. Device fabrication was partially carried out at the EPFL Center for Micro/ Nanotechnology (CMi). Special thanks to Ms. Anna Carlsson from FEI for the help in HRTEM imaging. The authors would like to thank Ulrich Keyser and Michael Walker for their helpful and constructive comments that greatly contributed to improving the quality of the paper. The work performed in 
Cambridge was supported by the EPSRC Cambridge NanoDTC, EP/L015978/1. The work performed in UIUC was supported by grants from Oxford Nanopore Technology and the Seeding Novel Interdisciplinary Research Program of the Beckman Institute. The UIUC authors gratefully acknowledge also supercomputer time provided through the Extreme Science and Engineering Discovery Environment (XSEDE) grant MCA93S028 and by the University of Illinois at UrbanaChampaign on the TAUB cluster. H.Q. acknowledges the computational support of the NSF China (11402113) and Jiangsu NSF (BK20140807).

\section{REFERENCES}

(1) Garaj, S.; Hubbard, W.; Reina, A.; Kong, J.; Branton, D.; Golovchenko, J. A. Nature 2010, 467, 190-193.

(2) Merchant, C. A.; Healy, K.; Wanunu, M.; Ray, V.; Peterman, N.; Bartel, J.; Fischbein, M. D.; Venta, K.; Luo, Z. T.; Johnson, A. T. C.; Drndic, M. Nano Lett. 2010, 10, 2915-2921.

(3) Schneider, G. F.; Kowalczyk, S. W.; Calado, V. E.; Pandraud, G.; Zandbergen, H. W.; Vandersypen, L. M. K.; Dekker, C. Nano Lett. 2010, 10, 3163-3167.

(4) Liu, K.; Feng, J. D.; Kis, A.; Radenovic, A. ACS Nano 2014, 8, 2504-2511.

(5) Feng, J. D.; Liu, K.; Graf, M.; Dumcenco, D.; Kis, A.; Di Ventra, M.; Radenovic, A. Nat. Mater. 2016, 15, 850-855.

(6) Gracheva, M. E.; Xiong, A. L.; Aksimentiev, A.; Schulten, K.; Timp, G.; Leburton, J. P. Nanotechnology 2006, 17, 622-633.

(7) Li, J.; Stein, D.; McMullan, C.; Branton, D.; Aziz, M. J.; Golovchenko, J. A. Nature 2001, 412, 166-169.

(8) Storm, A. J.; Chen, J. H.; Ling, X. S.; Zandbergen, H. W.; Dekker, C. Nat. Mater. 2003, 2, 537-540.

(9) Kim, M. J.; Wanunu, M.; Bell, D. C.; Meller, A. Adv. Mater. 2006, $18,3149-3153$.

(10) Kwok, H.; Briggs, K.; Tabard-Cossa, V. PLoS One 2014, 9, e92880.

(11) Kuan, A. T.; Lu, B.; Xie, P.; Szalay, T.; Golovchenko, J. A. Appl. Phys. Lett. 2015, 106, 203109.

(12) Feng, J.; Liu, K.; Graf, M.; Lihter, M.; Bulushev, R. D.; Dumcenco, D.; Alexander, D. T. L.; Krasnozhon, D.; Vuletic, T.; Kis, A.; Radenovic, A. Nano Lett. 2015, 15, 3431-3438.

(13) Alem, N.; Erni, R.; Kisielowski, C.; Rossell, M. D.; Gannett, W.;

Zettl, A. Phys. Rev. B: Condens. Matter Mater. Phys. 2009, 80, 155425.

(14) Jin, C. H.; Lin, F.; Suenaga, K.; Iijima, S. Phys. Rev. Lett. 2009, 102, 195505.

(15) Meyer, J. C.; Chuvilin, A.; Algara-Siller, G.; Biskupek, J.; Kaiser, U. Nano Lett. 2009, 9, 2683-2689.

(16) Hu, S.; Lozada-Hidalgo, M.; Wang, F. C.; Mishchenko, A.; Schedin, F.; Nair, R. R.; Hill, E. W.; Boukhvalov, D. W.; Katsnelson, M. I.; Dryfe, R. A. W.; Grigorieva, I. V.; Wu, H. A.; Geim, A. K. Nature 2014, 516, 227-230.

(17) Wanunu, M. Physics of Life Reviews 2012, 9, 125-158.

(18) Aguilella-Arzo, M.; Aguilella, V. M.; Eisenberg, R. S. Eur. Biophys. J. 2005, 34, 314-322.

(19) Branton, D.; Deamer, D. W.; Marziali, A.; Bayley, H.; Benner, S. A.; Butler, T.; Di Ventra, M.; Garaj, S.; Hibbs, A.; Huang, X. H.; Jovanovich, S. B.; Krstic, P. S.; Lindsay, S.; Ling, X. S. S.; Mastrangelo, C. H.; Meller, A.; Oliver, J. S.; Pershin, Y. V.; Ramsey, J. M.; Riehn, R.; Soni, G. V.; Tabard-Cossa, V.; Wanunu, M.; Wiggin, M.; Schloss, J. A. Nat. Biotechnol. 2008, 26, 1146-1153.

(20) Manrao, E. A.; Derrington, I. M.; Laszlo, A. H.; Langford, K. W.; Hopper, M. K.; Gillgren, N.; Pavlenok, M.; Niederweis, M.; Gundlach, J. H. Nat. Biotechnol. 2012, 30, 349-353.

(21) Feng, J. D.; Liu, K.; Bulushev, R. D.; Khlybov, S.; Dumcenco, D.; Kis, A.; Radenovic, A. Nat. Nanotechnol. 2015, 10, 1070-1076.

(22) Wanunu, M.; Dadosh, T.; Ray, V.; Jin, J. M.; McReynolds, L.; Drndic, M. Nat. Nanotechnol. 2010, 5, 807-814.

(23) Kowalczyk, S. W.; Grosberg, A. Y.; Rabin, Y.; Dekker, C. Nanotechnology 2011, 22, 315101.
(24) Caneva, S.; Weatherup, R. S.; Bayer, B. C.; Brennan, B.; Spencer, S. J.; Mingard, K.; Cabrero-Vilatela, A.; Baehtz, C.; Pollard, A. J.; Hofmann, S. Nano Lett. 2015, 15, 1867-1875.

(25) Caneva, S.; Weatherup, R. S.; Bayer, B. C.; Blume, R.; CabreroVilatela, A.; Braeuninger-Weimer, P.; Martin, M. B.; Wang, R. Z.; Baehtz, C.; Schloegl, R.; Meyer, J. C.; Hofmann, S. Nano Lett. 2016, $16,1250-1261$

(26) Tran, T. T.; Elbadawi, C.; Totonjian, D.; Lobo, C. J.; Grosso, G.; Moon, H.; Englund, D. R.; Ford, M. J.; Aharonovich, I.; Toth, M. ACS Nano 2016, 10, 7331-7338.

(27) Harrington, R. F. J. Electromagnetic Wave. 1987, 1, 181-200.

(28) Newman, E. H. IEEE Trans. Educ. 1988, 31, 193-200.

(29) Ryu, G. H.; Park, H. J.; Ryou, J.; Park, J.; Lee, J.; Kim, G.; Shin, H. S.; Bielawski, C. W.; Ruoff, R. S.; Hong, S.; Lee, Z. Nanoscale 2015, 7, 10600-10605.

(30) Elbadawi, C.; Tran, T. T.; Kolibal, M.; Sikola, T.; Scott, J.; Cai, Q. R.; Li, L. H.; Taniguchi, T.; Watanabe, K.; Toth, M.; Aharonovich, I.; Lobo, C. Nanoscale 2016, 8, 16182-16186.

(31) Pham, T.; Gibb, A. L.; Li, Z. L.; Gilbert, S. M.; Song, C. Y.; Louie, S. G.; Zettl, A. Nano Lett. 2016, 16, 7142-7147.

(32) Wong, D.; Velasco, J.; Ju, L.; Lee, J.; Kahn, S.; Tsai, H. Z.; Germany, C.; Taniguchi, T.; Watanabe, K.; Zettl, A.; Wang, F.; Crommie, M. F. Nat. Nanotechnol. 2015, 10, 949-953.

(33) Waduge, P.; Bilgin, I.; Larkin, J.; Henley, R. Y.; Goodfellow, K.; Graham, A. C.; Bell, D. C.; Vamivakas, N.; Kar, S.; Wanunu, M. ACS Nano 2015, 9, 7352-7359.

(34) Heerema, S. J.; Schneider, G. F.; Rozemuller, M.; Vicarelli, L.; Zandbergen, H. W.; Dekker, C. Nanotechnology 2015, 26, 074001.

(35) Wanunu, M.; Sutin, J.; McNally, B.; Chow, A.; Meller, A. Biophys. J. 2008, 95, 4716-4725.

(36) Mirsaidov, U.; Comer, J.; Dimitrov, V.; Aksimentiev, A.; Timp, G. Nanotechnology 2010, 21, 395501.

(37) Venta, K.; Shemer, G.; Puster, M.; Rodriguez-Manzo, J. A.; Balan, A.; Rosenstein, J. K.; Shepard, K.; Drndic, M. ACS Nano 2013, 7, 4629-4636.

(38) Sathe, C.; Zou, X. Q.; Leburton, J. P.; Schulten, K. ACS Nano 2011, 5, 8842-8851.

(39) Qiu, H.; Sarathy, A.; Leburton, J. P.; Schulten, K. Nano Lett. 2015, 15, 8322-8330.

(40) Hall, J. E. J. Gen. Physiol. 1975, 66, 531-532.

(41) Smeets, R. M. M.; Keyser, U. F.; Krapf, D.; Wu, M. Y.; Dekker, N. H.; Dekker, C. Nano Lett. 2006, 6, 89-95.

(42) Park, K. B.; Kim, H. J.; Kim, H. M.; Han, S. A.; Lee, K. H.; Kim, S. W.; Kim, K. B. Nanoscale 2016, 8, 5755-5763.

(43) Steinbock, L. J.; Bulushev, R. D.; Krishnan, S.; Raillon, C.; Radenovic, A. ACS Nano 2013, 7, 11255-11262.

(44) Rollings, R.; Graef, E.; Walsh, N.; Nandivada, S.; Benamara, M.; Li, J. Nanotechnology 2015, 26, 044001.

(45) Suk, M. E.; Aluru, N. R. J. Chem. Phys. 2014, 140, 084707.

(46) Girdhar, A.; Sathe, C.; Schulten, K.; Leburton, J. P. Proc. Natl. Acad. Sci. U. S. A. 2013, 110, 16748-16753.

(47) Qiu, H.; Sarathy, A.; Schulten, K.; Leburton, J.-P. npj $2 D$ Materials and Applications 2017, 1, 3.

(48) Heiranian, M.; Farimani, A. B.; Aluru, N. R. Nat. Commun. 2015, 6, 8616.

(49) Feng, J. D.; Graf, M.; Liu, K.; Ovchinnikov, D.; Dumcenco, D.; Heiranian, M.; Nandigana, V.; Aluru, N. R.; Kis, A.; Radenovic, A. Nature 2016, 536, 197-200. 